\title{
Video Article \\ 3D Imaging of Soft-Tissue Samples using an X-ray Specific Staining Method and Nanoscopic Computed Tomography
}

\author{
Madleen Busse ${ }^{{ }^{1}}$, Mark Müller* ${ }^{1}$, Melanie A. Kimm ${ }^{2}$, Simone Ferstl ${ }^{1}$, Sebastian Allner ${ }^{1}$, Klaus Achterhold ${ }^{1}$, Julia Herzen ${ }^{1}$, Franz Pfeiffer ${ }^{1,2}$ \\ ${ }^{1}$ Department of Physics and Munich School of BioEngineering, Technical University of Munich \\ ${ }^{2}$ Department of Diagnostic and Interventional Radiology, School of Medicine and Klinikum rechts der Isar, Technical University of Munich \\ * These authors contributed equally
}

Correspondence to: Madleen Busse at madleen.busse@tum.de

URL: https://www.jove.com/video/60251

DOI: doi:10.3791/60251

Keywords: Bioengineering, Issue 152, X-ray staining method, microscopic X-ray computed tomography, nanoscopic X-ray computed tomography, nondestructive, multiscale imaging, high-resolution, 3D imaging, soft tissue imaging, microstructure, eosin stain, cytoplasm-specific staining

Date Published: $10 / 24 / 2019$

Citation: Busse, M., Müller, M., Kimm, M.A., Ferstl, S., Allner, S., Achterhold, K., Herzen, J., Pfeiffer, F. 3D Imaging of Soft-Tissue Samples using an X-ray Specific Staining Method and Nanoscopic Computed Tomography. J. Vis. Exp. (152), e60251, doi:10.3791/60251 (2019).

\section{Abstract}

We demonstrate a laboratory-based method combining X-ray microCT and nanoCT with a specific X-ray stain, which targets the cell cytoplasm. The described protocol is easy to apply, fast and suitable for larger soft-tissue samples. The presented methodology enables the characterization of crucial tissue structures in three dimensions and is demonstrated on a whole mouse kidney. The multiscale approach allows to image the entire mouse kidney and supports the selection of further volumes of interest, which are acquired with higher resolutions ranging into the nanometer range. Thereby, soft-tissue morphology with a similar detail level as the corresponding histological light microscopy images is reproduced. Deeper insights into the 3D configuration of tissue structures are achieved without impeding further investigations through histological methods.

\section{Video Link}

The video component of this article can be found at https://www.jove.com/video/60251/

\section{Introduction}

Full characterization of soft-tissue specimens requires information about the 3D tissue microstructure. The current gold standard for soft-tissue sample analyses is histopathology. The tissue and cellular morphology of the specimen are explored in $2 \mathrm{D}$ within selected regions of interest (ROIs) using optical microscopy. This method, however, has several drawbacks. The preparation of the sample is time-consuming, complicated, destructive and prone to artifacts. The produced microscopic slides provide only $2 \mathrm{D}$ information parallel to the sectioning plane. Often the number of histological sections, which are investigated, is restricted due to time constraints ${ }^{2,3}$.

In recent years, the field of 3D histology has evolved. Here, virtual tissue slices from any desired spatial plane are accessible. This allows for the tracking of structures throughout the sample, which leads to a deeper understanding of the 3D tissue architecture and structural changes associated with different pathologies. Various methods have been developed to achieve the generation of 3D volume data. They range from serial-section based approaches, which use either light or electron microscopy ${ }^{4,5,6,7,8}$, to block-face imaging methods, such as episcopic 3D imaging or block-face scanning electron microscopy ${ }^{7,8,9}$. All the mentioned methods, however, involve either sectioning or destruct the sample completely, which does not allow for further investigations. The obtained resolution is highly dependent on the sectioning process being prone to artifacts as described in conventional histology. These methods suffer also from alignment artifacts.

3D X-ray imaging techniques such as microscopic and nanoscopic computed tomography (microCT and nanoCT) aspire to generate 3D highresolution data without destruction of the tissue sample. So far, the weak X-ray attenuation contrast of soft tissue and the limited access to high resolutions in a laboratory environment has impaired their use for 3D visualization of microscopic tissue structures. Recent advances towards laboratory-based, high-resolution X-ray CT allow for resolutions well below $1 \mu \mathrm{m}^{10,11,12,13}$.

The lack of contrast in soft tissue in conventional attenuation-based X-ray imaging is compensated by staining agents, which enhance the $X$ ray attenuation contrast. Staining agents known from other imaging techniques such as osmium tetroxide $\left(\mathrm{OsO}_{4}\right)$, iodine potassium iodide (IKI) or phosphotungstic acid (PTA) are often used ${ }^{14,15,16,17,18,19,20,21,22,23,24,25}$. Staining agents that allow for (i) specific biological targeting, (ii) homogenous and complete staining, (iii) easy handling, (iv) fast penetration of the tissue without creating artifacts such as diffusion rings, ( $v$ ) large and dense tissue staining, and (vi) full compatibility with histopathology are required to establish X-ray CT as tool for 3D visualization of microscopic tissue structures. In this work, we show how soft-tissue samples are prepared for X-ray CT imaging with a cytoplasm-specific X-ray stain based on eosin that fulfills the requirements stated above ${ }^{26}$.

The multiscale imaging approach ensures the assessment of staining quality through an overview microCT measurement and the selection of volumes of interest (VOIs) for further high-resolution investigations. Staining quality is analyzed focusing on staining parameters such as (i) 
completeness, (ii) appearance of diffusion rings, (iii) contrast enhancement, (iv) appearance of CT artifacts such as streaks and (v) homogeneity. The laboratory-based nanoCT setup, which uses geometric magnification to reach resolutions down to $100 \mathrm{~nm}$, visualizes soft-tissue morphology on (sub)-cellular level ${ }^{10,27}$. A comparative analysis of the nanoCT slices with corresponding histological light microscopy images confirms the reproduction of tissue architecture with similar detail on a microscopic level in $2 \mathrm{D}$, enabling histopathological characterization of the tissue sample. This detailed video protocol is intended to raise the awareness and to highlight the potential of this methodology as non-destructive 3D soft-tissue imaging tool being of interest to a wide scientific community such as zoologists, biologists and health professionals.

\section{Protocol}

Caution: Please consult all relevant material safety data sheets (MSDS) before use. Several of the chemicals used in the protocol are acutely toxic and carcinogenic. Please use all appropriate safety practices when performing the staining protocol including the use of engineering controls (fume hood, glovebox) and personal protective equipment (safety glasses, gloves, lab coat, full length pants, closed-toe shoes).

\section{Animals Used:}

Animal housing was carried out at the Klinikum rechts der Isar, Technical University of Munich in accordance with the European Union guidelines 2010/63. Organ removal was approved from an internal animal protection committee of Klinikum rechts der Isar, Munich, Germany (internal reference number 4-005-09). All procedures were in accordance with relevant guidelines and regulations. All laboratories are inspected for accordance with the OECD principles of good laboratory practice.

\section{Eosin staining protocol}

1. To fixate soft-tissue samples, fill a $50-\mathrm{mL}$ centrifuge tube with a fixative solution containing $9.5 \mathrm{~mL}$ of $4 \%$ ( $/ \mathrm{v}$ ) formaldehyde solution (FA) and $0.5 \mathrm{~mL}$ of glacial acetic acid (AA).

NOTE: Prepare the FA solution freshly from a $37 \%$ acid free FA solution stabilized with approximate $10 \%$ methanol. Dilute the FA solution further with Dulbecco's phosphate buffered saline (DPBS). Choose DPBS without calcium and magnesium. Keep the dilute FA solution no longer than one month. During acidification the $\mathrm{pH}$ of the fixative solution is changing from neutral to approximately 3 .

CAUTION: Because FA is acute organ toxic, corrosive and carcinogenic, the use of a fume hood is mandatory and appropriate protective personal equipment must be used.

1. Add the freshly removed soft-tissue sample to a $50-\mathrm{mL}$ centrifuge tube and refrigerate the $50-\mathrm{mL}$ centrifuge tube for $24-72 \mathrm{~h}$. NOTE: The protocol can be paused here.

2. Wash the soft-tissue sample with DPBS solution for $1 \mathrm{~h}$.

2. To stain the fixated soft-tissue sample (e.g., a whole mouse kidney), place the soft-tissue in $2 \mathrm{~mL}$ of eosin Y-staining solution and incubate the sample for $24 \mathrm{~h}$. Keep the sample on a horizontal shaking plate for a smooth rocking (ca. $60 \mathrm{rpm}$ ) during the incubation process.

NOTE: The eosin Y-staining solution has a concentration of $30 \%(\mathrm{w} / \mathrm{v})$ in distilled water. Choose the volume of the staining solution in such a way that the sample is completely covered by the staining solution and allow the sample to move freely within the sample container. The incubation time may differ for other samples and has to be adjusted accordingly.

3. After staining, remove the soft-tissue sample carefully from the sample container.

1. Carefully remove excess of staining agent with cellulose tissue paper.

2. Place the soft-tissue sample in a conical sample container above an ethanol vapor phase for storage and further use. NOTE: The conical sample container must always contain a few drops of $70 \%(\mathrm{v} / \mathrm{v})$ ethanol at the bottom of the tube to keep the softtissue sample moist and prevent artifacts.

\section{X-ray microCT imaging}

NOTE: The X-ray microCT measurements were performed with a microCT scanner, which offers overview CT measurements (the ability to image the entire sample within the field of view (FOV)) and the performance of high-resolution CT measurements (the ability to focus in on one desired volume of interest (VOI) of the very same sample) down to $1 \mu \mathrm{m}$.

1. Mount the soft-tissue sample to an appropriate sample holder. Ensure a tight fit of the sample on the sample holder to prevent the sample from moving during the X-ray CT measurements.

1. In case of the stained mouse kidney: Prepare a sample holder with two centrifuge tubes, whereby the bottom of one tube is cut off. Glue the two centrifuge tubes together using two-component adhesive. Ensure a straight alignment of the centrifuge tubes around the rotation axis. Wait for the adhesive to harden.

2. Once the sample holder is ready for use, transfer the mouse kidney into the intact centrifuge tube, which holds a few drops of $70 \%(\mathrm{v} / \mathrm{v})$ ethanol at the bottom of the tube.

NOTE: Stability of the sample is crucial. Take time to prepare the sample for X-ray CT measurements. The soft-tissue sample is kept over an ethanol vapor phase to keep the sample moist during the X-ray CT measurements and prevent the soft-tissue sample from shrinkage and other artifacts. The soft-tissue sample should not be in contact with the solvent to obviate accumulation of the solvent around the sample during X-ray CT measurement, which might lead to sample movement during the measurement or might cause problems during reconstruction. If the sample holder does not allow to hold solvent at the bottom, a cellulose paper moistened with $70 \%(\mathrm{v} / \mathrm{v})$ ethanol can be placed in the sample holder. It should be noted that shrinkage artifacts due to the solvent ethanol were not observed.

NOTE: The protocol can be paused here.

2. After careful alignment of the sample, choose acquisition parameters for best image quality. In case of the presented microCT data, acquire the scan at a peak voltage of $50 \mathrm{kV}$, a current of $3.5 \mathrm{~W}$ using 1601 projections equally distributed over $360^{\circ}$. 
NOTE: The acquisition parameters for the overview CT scan were chosen for best image quality. As such the 0.39x camera objective was chosen to cover the whole sample within the field of view (FOV). This resulted in an effective pixel size of $12 \mu \mathrm{m}$. The exposure time of $2 \mathrm{~s}$ per projection provided a good signal to noise ratio. The ROI for the high-resolution CT scan was identified using the microCT data from the overview scan. MicroCT scanners often incorporate an integrated software tool, which allows for the precise selection of the determined ROI. For the high-resolution CT data, the $4 x$ camera objective was chosen resulting in an effective pixel size of $3.3 \mu \mathrm{m}$. Here, an exposure time of $15 \mathrm{~s}$ per projection was needed.

NOTE: The protocol can be paused here.

3. After acquisition of the X-ray CT data, process the projections accordingly for reconstruction of the $3 \mathrm{D}$ volume. In case of the presented microCT data: Reconstruct the X-ray CT data with the integrated software.

NOTE: The volume renderings of the microCT data shown in Figure 1 and Figure 2 were generated using a visualization software. NOTE: The protocol can be paused here.

\section{X-ray nanoCT imaging}

NOTE: The X-ray nanoCT scanner has been developed inhouse. The lens free instrument is equipped with a nanofocus X-ray source and a single-photon counting detector. 3D data with resolutions down to $100 \mathrm{~nm}$ can be generated ${ }^{10}$. Generally, nanoCT systems including those with $\mathrm{X}$-ray optics are commercially available and not limited to the described nanoCT scanner.

1. NanoCT sample preparation

1. Prepare VOls of the soft-tissue sample. Cut the soft tissue into very small pieces of approximately $0.5 \mathrm{~mm}$ edge length using a scalpel and a stereomicroscope. In case of the mouse kidney: Cut the mouse kidney into two halves along the longest axis. Take one half of the mouse kidney and prepare different anatomical regions such as renal cortex and renal medulla.

NOTE: The other halve of the mouse kidney was transferred to histopathology, where the sample was embedded into paraffin and processed accordingly to yield the typical histological sections as seen in Figure 3c and Figure 3d.

2. Transfer the small pieces before the first dehydration step to a new Petri dish, where they remain for all subsequent steps.

3. Dehydrate the samples using concentrations (all v/v) in \%: 50, 60, 70, 80, 90, 96 and 100 ethanol balanced with distilled water. Perform each dehydration step for $1 \mathrm{~h}$ each.

NOTE: The protocol can be paused here. Keep the small tissue pieces in $100 \%$ ethanol overnight.

4. Critical point dry (CPD) the small tissue pieces.

NOTE: The application of CPD enables the complete dehydration of the tissue sample by exchanging the solvent (here ethanol) with the drying agent (here $\mathrm{CO}_{2}$ ). This has been necessary to ensure that the sample can be mounted to the sample holders of the nanoCT, is not moving during the measurement and can be positioned very close to the X-ray source to allow for best geometrical magnification. The nanoCT setup is based on mere geometrical magnification, with the magnification factor being defined as the source-to-detector distance over the source-to-sample distance. The drying technique was first introduced by Anderson to preserve the 3D structure of biological specimens for electron microscopy ${ }^{28}$. An overview of the technique is provided by Bray ${ }^{29}$.

1. Prefill the vacuum chamber with $100 \%$ ethanol. Transfer the small tissue pieces into a micro-porous capsule and place it in the vacuum chamber of the CPD. Close the system.

NOTE: Since high pressure is involved in the CPD process, ensure all parts of the CPD, in particular the fittings, are intact and the system is properly closed.

2. Cool the chamber to $6-8^{\circ} \mathrm{C}$ and fill up with liquid $\mathrm{CO}_{2}$.

3. While stirring, wait $3 \mathrm{~min}$ to allow for proper mixing of the two components. Carefully drain the chamber. Ensure the sample holder is still covered with solvent. Repeat this step ten times to allow complete replacement of ethanol with $\mathrm{CO}_{2}$ within the sample.

4. After the final filling of the chamber with $\mathrm{CO}_{2}$, heat the machine to the critical point of $\mathrm{CO}_{2}\left(31^{\circ} \mathrm{C}\right.$ and 73.8 bar $)$ followed by very slow release of the gaseous $\mathrm{CO}_{2}$ over a time of $30 \mathrm{~min}$.

NOTE: The release of the gas should be undertaken very slowly as otherwise condense water can form on the sample. Ensure that the temperature does not drop below the critical point of $\mathrm{CO}_{2}$. Only open the CPD machine when all pressure has been released from the system.

5. Remove the CPD tissue pieces quickly from the machine and keep them in a new Petri dish stored in a desiccator prior to further use.

NOTE: The protocol can be paused here.

2. Mount the CPD tissue pieces to an appropriate sample holder. Ensure a tight fit of the sample on the sample holder to prevent the sample from moving during the CT measurements. In case of the CPD mouse kidney tissue pieces: Glue the tissue pieces with superglue to a sample holder.

NOTE: Any undesired movement of the sample during CT measurements can cause problems during volume reconstruction - especially when acquiring a data set with nanometer voxel size.

NOTE: The protocol can be paused here.

3. After careful alignment of the sample, choose acquisition parameters for best image quality. In case of the presented nanoCT data: Acquire projections at a peak voltage of $60 \mathrm{kV}$ with 1599 projections equally distributed over $360^{\circ}$ and a voxel size of approximately $400 \mathrm{~nm}$. NOTE: A single CT measurement acquired at $400 \mathrm{~nm}$ voxel size has a FOV of $75 \mu \mathrm{m}$ in the direction of the rotation axis (vertical) and approximately $560 \mu \mathrm{m}$ in the direction perpendicular to the rotation axis (horizontal). To investigate larger volumes, an extension of the FOV along the rotation axis can be achieved by combining multiple scans at different vertical positions. Additionally, local tomography scans can be performed to measure samples with a larger sample diameter perpendicular to the rotation axis than given by the FOV of a global CT scan. The nanoCT data was acquired with an exposure time of $4 \mathrm{~s}$ per projection. As such the total acquisition time per data set was approximately $3.5 \mathrm{~h}$.

NOTE: The protocol can be paused here.

4. After acquisition of the CT data, process the projections accordingly for reconstruction of the 3D volume. 
1. In case of the presented nanoCT data, normalize the acquired projections with flatfield images. Enhance the sharpness of the projections by using a Richardson-Lucy deconvolution algorithm ${ }^{30,31}$. Use a rotationally symmetric Gaussian function with a standard deviation of one pixel as deconvolution kernel.

2. Apply Paganin's phase-retrieval algorithm to the sharpened images to increase the soft-tissue contrast. Set the parameters of the algorithm to optimize the image quality ${ }^{32}$. Reconstruct the preprocessed projections with a state-of-the-art filtered backprojection algorithm.

NOTE: Figure 3 evaluates the obtained nanoCT data with corresponding histological sections, which were approximately $7 \mu \mathrm{m}$ thick. Therefore, minimum intensity projection slices of 18 adjacent nanoCT slices with a virtual thickness of approximately $7 \mu \mathrm{m}$ were generated by means of calculating the minimum value for each pixel in the relevant slices. A visualization software was used to render the volume of the nanoCT data, which is displayed in Figure 4.

\section{Representative Results}

Figure 1 shows CT slices and volume rendering of low-resolution microCT data highlighting contrast enhancement after staining. Figure 2 shows CT slices and volume rendering of high-resolution microCT data derived from a local tomography of the whole mouse kidney. Figure 3 shows CT slices of nanoCT data in comparison to the corresponding histological sections. Figure 4 shows CT slice and volume rendering of nanoCT data highlighting structural details at cellular level. The low-resolution microCT measurement allows for an overview of the whole organ and helps to identify volumes of interest (VOIs) for the high-resolution microCT measurement. Through this multiscale approach, the VOI for the nanoCT is determined. The nanoCT enables a very detailed view of the soft-tissue sample on cellular level. The comparative study with the corresponding histological section highlights full compatibility with histopathology. Here, the multimodal imaging approach is confirming the results obtained with both modalities.
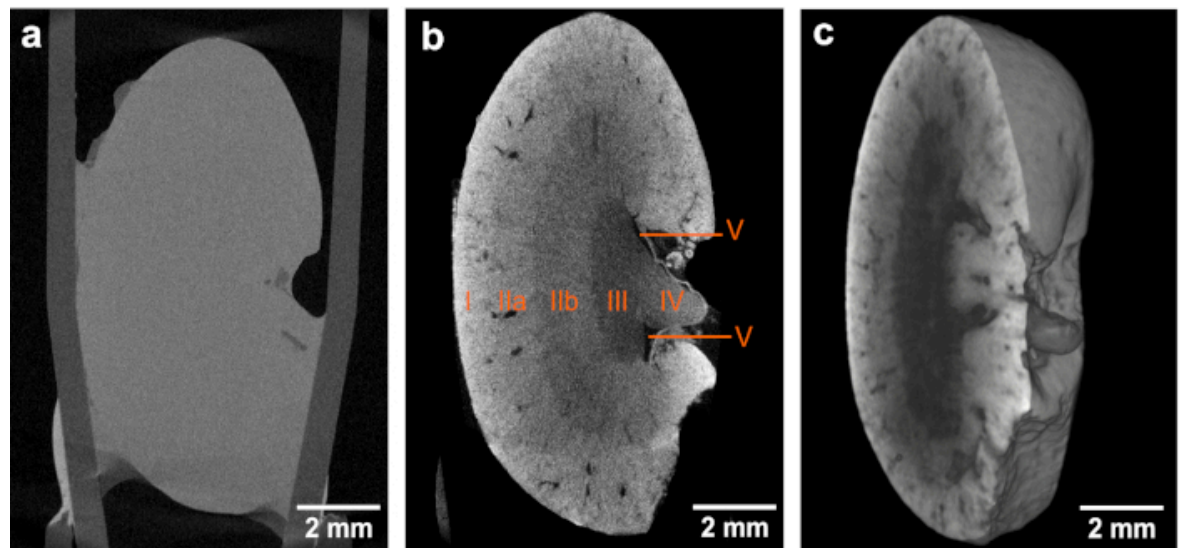

Figure 1. CT slices and volume rendering of the low-resolution microCT data. (a,b) Overview images of the same mouse kidney before and after staining, respectively, highlighting the contrast enhancement obtained after application of the eosin-based staining protocol. Both microCT data sets were acquired using identical acquisition parameters. The voxel size in both data sets is $12 \mu \mathrm{m}$. The contrast enhancement achieved in (b) enables the identification of the following anatomic structural regions: Cortex (I), outer medulla (II) with further distinction in outer stripes of outer medulla (Ila) and inner stripes of outer medulla (Ilb), inner medulla (III), papilla (IV) and renal pelvis (V). (c) Volume rendering of microCT data showing a virtual sagittal section through the whole mouse kidney. This figure has been modified from Busse and Müller et al. ${ }^{26}$ Please click here to view a larger version of this figure. 

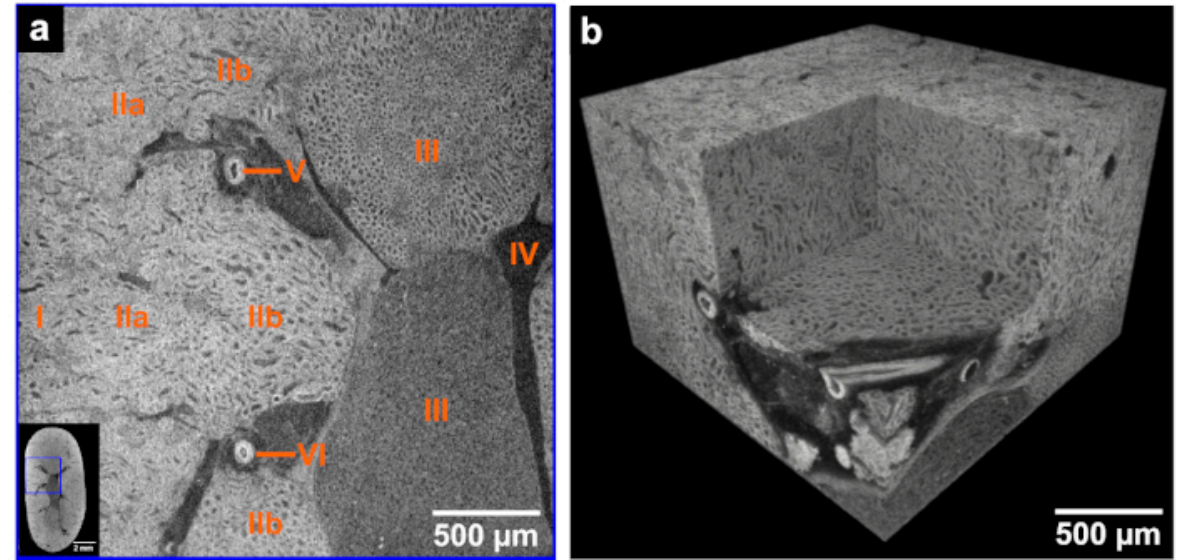

Figure 2. CT slice and volume rendering of high-resolution microCT data derived from the same mouse kidney after application of the developed eosin-based staining protocol. (a) The left corner shows the overview microCT image highlighting the ROI (blue box) for the displayed high-resolution image. The following anatomic structural regions are identifiable: Cortex (I), outer medulla (II) with further distinction in outer stripes of outer medulla (Ila) and inner stripes of outer medulla (IIb), inner medulla (III), minor calyx (IV) and vessels (V and VI). (b) Volume of interest rendering of the high-resolution microCT data acquired with a voxel size of $3.3 \mu \mathrm{m}$. The medulla region and a virtual section through a vessel derived from a local tomography of the whole kidney is shown. This figure has been modified from Busse and Müller et al. ${ }^{26}$. Please click here to view a larger version of this figure.
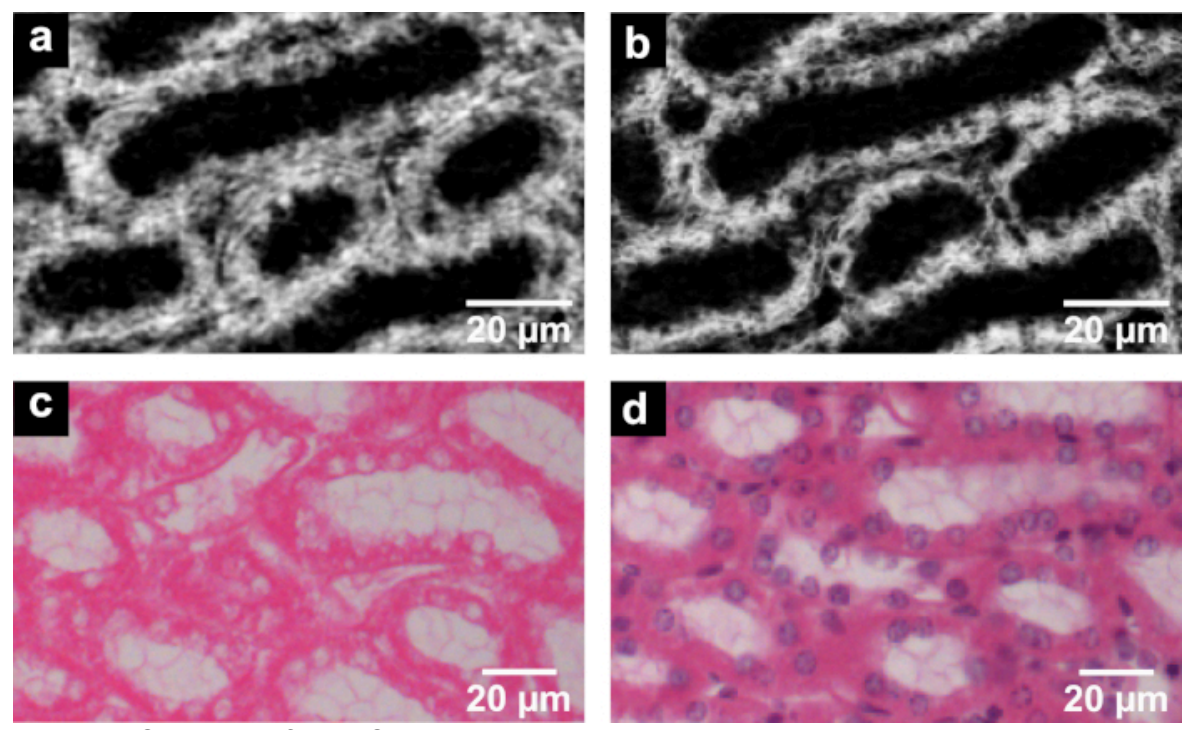

Figure 3. CT slices of nanoCT data $(a, b)$ in comparison to the histological sections (c,d) derived from the same mouse kidney after application of the developed eosin-based staining protocol. (a) The nanoCT image of the same mouse kidney sample after staining, dissecting and CPD shows detailed structures of region (IIb) seen in Figure 1 and Figure 2. These are known as thick ascending limbs of the loop of Henle. (b) Minimum intensity projection slice derived from the same nanoCT data set shown in (a) with a virtual slice thickness of approximately $7 \mu \mathrm{m}$, which allows for clear visualization of the cell nuclei. (c) Representative histological section displaying thick ascending limbs of the loop of Henle with clear visualization of cell nuclei and brush border. The histological section has an approximate thickness of $7 \mu \mathrm{m}$ and was obtained from the same mouse kidney sample after the applied eosin-based staining and embedding in a paraffin block. (d) Representative histological section with application of counter stain hematoxylin highlighting the cell nuclei in purple. Preparation of the histological section close to the section shown in (c) with approximate thickness of $7 \mu \mathrm{m}$. This figure has been modified from Busse and Müller et al. ${ }^{26}$ Please click here to view a larger version of this figure. 

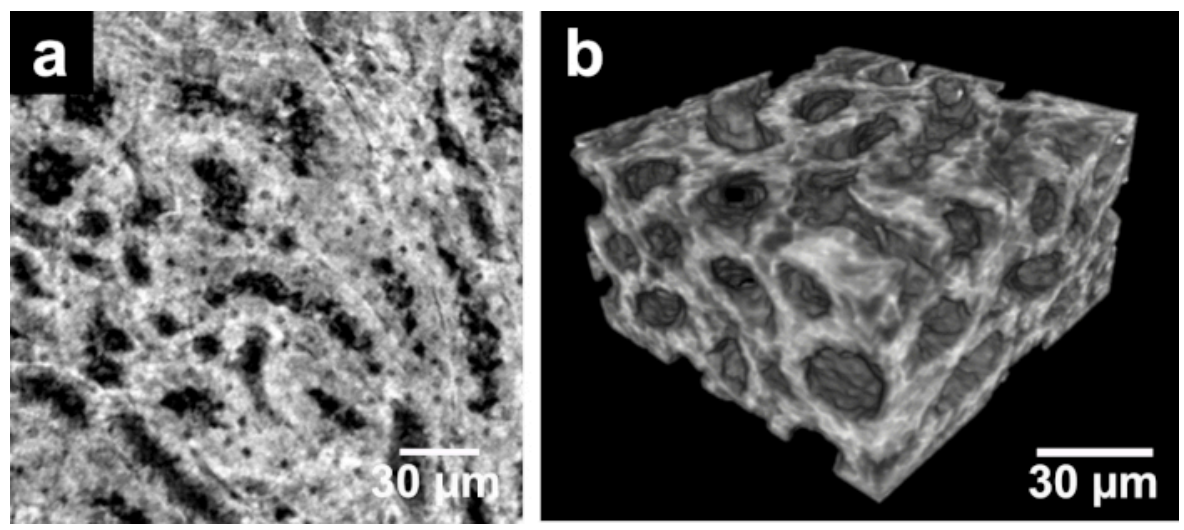

Figure 4. CT slice and volume rendering of nanoCT data. (a) The nanoCT image of the same mouse kidney sample showing the structures known as thick ascending limbs of the loop of Henle. This is a detailed view of region (Ilb) seen in Figure $\mathbf{1}$ and Figure $\mathbf{2}$ acquired from a small piece of the kidney with a voxel size of approximately $400 \mathrm{~nm}$. The preparation of the sample involved staining, dissecting and CPD. (b) Volume rendering of nanoCT data visualizing the 3D structure of thick ascending limbs of the loops of Henle. This figure has been modified from Busse and Müller et al. ${ }^{26}$ Please click here to view a larger version of this figure.

\section{Discussion}

Currently, eosin is used as the standard histological protocol to label the cell cytoplasm. The staining agent is applied as a $0.1 \%$ (w/v) aqueous solution to microscopic slices of soft tissue (generally cut with a thickness of 2-10 $\mu \mathrm{m})^{33}$. The application of this standardized histological protocol to $3 \mathrm{D}$ tissue samples such as a whole mouse kidney does not result in an attenuation contrast enhanced CT image. On the one hand, this can be attributed to the low intrinsic attenuation properties of soft tissue for typically used X-ray energies of laboratory-based microCT systems. Usually, soft tissue is composed of mainly carbon, hydrogen, oxygen and nitrogen ${ }^{34}$, and therefore, does not result in contrast enhancement. On the other hand, the low concentration of eosin used for staining was the limiting factor. Even though one eosin molecule holds four bromide atoms (high atomic number element bromine with $Z=35^{34}$ ), the sensitivity levels required for $X$-ray CT imaging were not met.

To overcome this challenge of low attenuation contrast, several concentrations of eosin were investigated. A limitation is here the maximum solubility of eosin in water, which is $30 \%(\mathrm{w} / \mathrm{v})$ in an aqueous solution. Best attenuation contrast enhancement within the soft tissue was observed with the highest eosin concentration, which was expected according to the Lambert-Beer Law. Therefore, the final staining protocol was carried out with the highest concentration.

The question how to prepare the soft tissue optimally on a molecular level for the staining procedure to further improve contrast enhancement was answered by $\mathrm{pH}$ adjustment. Here, the acidification of the soft-tissue sample during fixation or before staining was found to be crucial. This was also shown by Hong et al. ${ }^{35}$. The higher accumulation of staining agent within the cell cytoplasm by the acid was achieved through improved ionic interactions, which were a result of the protonation of amino acid side chains of proteins and peptides present within the cell cytoplasm. A representative result highlighting the contrast enhancement in comparison to an unstained soft-tissue sample is shown in Figure 1a,b. Here, a structural overview of a whole mouse kidney visualizing crucial anatomical regions such as cortex, medulla, papilla and renal pelvis was achieved.

The presented staining protocol is simple to apply and contains only three steps. The required reagents are easily accessible. The overall staining time of 24 hours is fast for a whole-organ staining, which enables the 3D visualization of soft-tissue samples (Figure 1c, Figure $\mathbf{2 b}$ and Figure $4 b$ ) in a laboratory environment at multiple scales down to cellular level. It should be noted that the overall staining time and volume of the staining solution needed might request some adaptations depending on the nature of the sample. Nevertheless, the eosin-based staining protocol is suitable for whole-organ staining, which then enables high-resolution microCT imaging of whole organs. Shrinkage artifacts due to the solvent ethanol, which was used to keep the sample moist during the microCT measurements, were not observed. Additional preparation steps are required for nanoCT imaging, which allows for the investigation of smaller tissue pieces retrieved from the original sample. With respect to future histopathological applications, the overview scans will provide valuable insights into altered anatomical regions and structures, which allow for the determination of ROls as demonstrated in Figure 2a. Those can be studied in 3D by microCT (Figure 1c and Figure $\mathbf{2 b}$ ) or nanoCT (Figure 4b) and evaluated in 2D with histology (Figure 3).

Another strength of the protocol is seen in the full compatibility with histopathology with respect to the H\&E staining procedure. The application of the eosin-based staining procedure to bulk samples does not impede further histological investigations (Figure 3), even though the applied eosin concentration is much higher compared to the histological staining solution. The nanoCT slice with a virtual thickness of approximately $400 \mathrm{~nm}$ (Figure 3a) compares already very well with the histological section (Figure 3c), which was derived from the corresponding soft-tissue sample. Considering the approximate thickness of a histological section with 7-10 $\mu \mathrm{m}$, the generation of minimum intensity projection slices of the nanoCT data (Figure 3b), which correspond to a virtual thickness of approximately $7 \mu \mathrm{m}$, allows for a better comparison with the histological section (Figure 3c). Here, the cell nuclei are clearly revealed as non-attenuation area as eosin specifically stains proteins and peptides in the cell cytoplasm $^{33}$.

The application of further counter staining with standard histological methods is possible, even though the order of the staining compared to the standard histological staining procedure was reversed. Starting first with the developed eosin-based staining protocol for CT, followed by counter staining of those eosin-based histological sections with hematoxylin, allows for full compatibility and results in a high-quality staining displaying the expected form of appearance. The cell nuclei-specific staining with Mayer's sour hematoxylin was applied to the histological section highlighting the cell nuclei in purple (Figure 3d). The application of histological counter staining is currently limited to the $\mathrm{H}$-stain. Other 
standard histological counter stainings such as periodic acid Schiff's base, Elastica van Gieson or Gomori silver have to be evaluated as well as the compatibility with immunohistological techniques needs to be tested.

The eosin-based staining protocol allows for (i) cell cytoplasm-specific targeting, (ii) homogenous and complete staining, (iii) easy implementation, (iv) fast penetration of the tissue without creating artifacts such as diffusion rings, (v) the staining of large and dense soft-tissue samples, and (vi) full compatibility with histopathology in respect of the H\&E stain. These requirements are important to allow high-resolution X-ray CT visualization of soft tissue down to cellular level. In combination with the recently developed nanoCT devices ${ }^{12,36,37}$, nondestructive generation of virtual histological slices that are comparable in contrast and resolution to conventional histological data is rendered possible. This combined approach will enable the establishment of X-ray CT as a valuable tool for the 3D visualization of microscopic tissue structures

\section{Disclosures}

The authors have nothing to disclose.

\section{Acknowledgments}

We thank Dr. Enken Drecoll for histological discussions and the extremely helpful team at Excillum AB, Sweden. We acknowledge financial support through the DFG Cluster of Excellence Munich Center for Advanced Photonics (MAP) and the DFG Gottfried Wilhelm Leibniz Program. Furthermore, this research project has received funding from the European's Union Horizon 2020 research and innovation program under the Marie Skłodowska-Curie Grant Agreement No. H2020-MSCA-IF-2015-703745-CONSALT.

\section{References}

1. Suvarna, S. K., Layton, C., Bancroft, J. D. Theory and Practice of Histological Techniques. 7th edn, Churchill Livingstone Elsevier, (2013).

2. Chatterjee, S. Artefacts in histopathology. Journal of Oral and Maxillofacial Pathology. 18 (4), 111-116 (2014).

3. McInnes, E. Artefacts in histopathology. Comparative Clinical Pathology. 13 (3), 100-108 (2005).

4. Andreasen, A., Drewes, A., Assentoft, J., Larsen, N. Computer-assisted alignment of standard serial sections without use of artificial land-marks. A practical approach to the utilization of incomplete information in 3-d reconstruction of the hippocampal region. Journal of Neuroscience Methods. 45 (3), 199-207 (1992).

5. Braverman, M. S., Braverman, I. M. Three-dimensional reconstructions of objects from serial sections using a microcomputer graphics system. Journal of Investigative Dermatology. 86 (3), 290-294 (1986).

6. Denk, W., Hortsmann, H. Serial block-face scanning electron microscopy to reconstruct three-dimensional tissue nanostructure PLoS Biology 2 (11), e329 (2004).

7. Mohun, T. J., Weninger, J. W. Imaging heart development using high-resolution episcopic microscopy. Current Opinion in Genetics and Development. 21, 573-578 (2011).

8. Weninger, J. W., Meng, S., Streicher, J., Müller, G. B. A new episcopic method for rapid 3-d reconstruction: applications in anatomy and embryology. Anatomy and Embryology. 197 (5), 341-348 (1998).

9. Odgaard, A., Andersen, K., Melsen, F., Gundersen, H. J. G. A Direct Method for Fast 3-Dimensional Serial Reconstruction. Journal of Microscopy. 159, 335-342 (1990).

10. Müller, M. et al. Myoanatomy of the velevt worm leg revealed by labratory-based nanofocus X-ray source tomography. Proceedings of the National Academy of Sciences of the United States of America. 114 (47), 12378-12383 (2017).

11. Salomon, M., Hanke, R., Krüger, P., Uhlmann, N., Voland, V. Realization of a computed tomography setup to achieve resolutions below $1 \mu$ m. Nuclear Instruments and Methods in Physics Research Section A. 591, 50-53 (2008).

12. Tkachuk, A. et al. X-ray computed tomography in Zernike phase contrast mode at $8 \mathrm{keV}$ with 50 -nm resolution using Cu rotating anode $\mathrm{X}$-ray source. Zeitschrift für Kristallographie. 222, 650-655 (2007).

13. Withers, P. J. X-ray nanotomography. Materials Today. 10, 26-34 (2007).

14. Jahn, $\mathrm{H}$. et al. Evaluation of contrasting techniques for X-ray imaging of velvet worms (Onychophora). Journal of Microscopy. 270 (3), 343-358 (2018).

15. Martins de S. e Silva, J. et al. Three-dimensional non-destructive soft-tissue visualization with X-ray staining micro-tomography. Scientific Reports. 5, 14088 (2015).

16. Metscher, B. D. MicroCT for Developmental Biology: A Versatile Tool for High-Contrast 3D Imaging at Histological Resolutions. Developmental Dynamics. 238, 632-640 (2009)

17. Metscher, B. D. MicroCT for comparative morphology: simple staining methods allow high-contrast $3 \mathrm{D}$ imaging of diverse non-mineralized animal tissues. BMC Physiology. 9, 11 (2009).

18. Mitzutani, R. et al. X-Ray Microtomographic Imaging of Three-Dimensional Structure of Soft Tissues. Tissue Engineering Part C: Methods. 14 (4), 359-363 (2008).

19. Pauwels, E., Loo, D. v., Cornillie, P., Brabant, L., Hoorebeke, L. v. An exploratory study of contrast agents for soft tissue visualization by means of high resolution X-ray computed tomography imaging. Journal of Microscopy. 250, 21-31 (2013).

20. Degenhardt, K., Wright, A. C., Horng, D., Padmanabhan, A., Epstein, J. A. Rapid 3D phenotyping of cardiovascular development in mouse embryos by micro-CT with iodine staining. Circulation: Cardiovascular Imaging. 3 (3), 314-322 (2010).

21. Dullin, $C$. et al. $\mu C T$ of ex-vivo stained mouse hearts and embryos enables a precise match between $3 \mathrm{D}$ virtual histology, classical histology and immunochemistry. PLOS ONE. 12 (2), e0170597 (2017).

22. Jeffrey, N. S., Stephenson, R. S., Gallagher, J. A., Cox, P. Micro-computed tomography with iodine staining resolves the arrangement of muscle fibres. Journal of Biomechanics. 44, 189-192 (2011).

23. Johnson, J. T. et al. Virtual Histology of Transgenic Mouse Embryos for High-Throughput Phenotyping. PLOS Genetics. 2, e61 (2006).

24. Leszczyński, B. et al. Visualization and Quantitative 3D Analysis of Intraocular Melanoma and Its Vascularization in a Hamster Eye. International Journal of Molecular Sciences. 19 (2), 332 (2018). 
25. Mizutani, R., Suzuki, Y. X-ray microtomography in biology. Mircon. 43, 104-115 (2012).

26. Busse, M. et al. Three-dimensional virtual histology enabled through cytoplasm-specific X-ray stain for microscopic and nanoscopic computed tomography. Proceedings of the National Academy of Sciences of the United States of America. 115 (10), 2293-2298 (2018).

27. Müller, M. et al. Non-destructive high-resolution 3D virtual histology enabled through a cell nucleus-specific stain for X-ray computed tomography. Scientific Reports. 8, 17855 (2018).

28. Anderson, T. F. Techniques for the preservation of three-dimensional structure in preparing specimens for the electron microscope. Transactions of the New York Academy of Sciences. 13 (4 Series II), 130-134 (1951).

29. Bray, D. in Supercritical Fluid Methods and Protocols. Methods in Biotechnology. Vol. 13 eds J. R. Williams, A. A. Clifford, Humana Press. 235-243 (2000).

30. Lucy, L. B. An iterative technique for the rectification of observed distributions. The Astronomical Journal. 79, 745-765 (1974).

31. Richardson, W. H. Bayesian-Based Iterative Method of Image Restoration. The Journal of the Optical Society of America. 62 (1), 55-59 (1972).

32. Paganin, F., Mayo, S. C., Gureyev, T. E., Miller, P. R., Wilkins, S. W. Simultaneous phase and amplitude extraction from a single defocused image of a homogeneous object. Journal of Microscopy. 206, 33-40 (2002).

33. Riedelsheimer, B., Büchl-Zimmermann, S. in Mikroskopische Technik 10.1007/978-3-642-55190-1 eds M. Mulisch \& U. Welsch Ch. 10, 193-194 (Spektrum-Verlag GmbH Berlin Heidelberg). (2015).

34. Hubbell, J. H., Seltzer, S. M. Tables of Xray mass attenuation coefficients and mass energy-absorption coefficients from $1 \mathrm{keV}$ to $92 \mathrm{keV}$ and 48 additional substances of dosimetric interest, Table 3. National Institute of Standards and Technology. NISTIR 5632 (1995).

35. Hong, H. Y., Yoo, G. S., Choi, J. K. An Eosin Y Method for Protein Determination in Solution. Analytical Letters. 32 (12), $2427-2442$ (1999).

36. Dierick, M. et al. Recent Micro-CT Scanner Developments at UGCT. Nuclear Instruments and Methods in Physics Research Section B. 324, 35-40 (2014).

37. Kastner, J., Plank, B., Heinzl, C. Advanced X ray computed tomography methods: High resolution CT, quantitative CT, 4DCT and phase contrast CT in Proceedings of Digital Industrial Radiology and Computed Tomography. 120-132 (2015). 